\title{
APLICACIÓN DE UN PROGRAMA DE CÁLCULO TÉRMICO PARA COMPROBAR EL FUNCIONAMIENTO DE UN MOTOR DIESEL PERKINS MODELO C4.236.V - GASODIESEL MEDIANTE ANÁLISIS COMPARATIVO
}

\author{
APPLICATION OF THERMAL CALCULATION PROGRAM TO \\ CHECK THE OPERATION OF A MOTOR PERKINS DIESEL \\ MODEL C4.236.V - COMPARATIVE ANALYSIS BY GASODIESEL
}

\author{
Maza Rubina Arturo모 Lastra Espinoza Luis ${ }^{2}$
}

\begin{abstract}
RESUMEN
Este trabajo de investigación, se realizó en la ciudad de Lima y trata, sobre el desarrollo y aplicación de un programa en base al cálculo térmico; para determinar los parámetros de funcionamiento del motor de combustión interna de uso automotriz mediante la simulación de los distintos procesos que tienen lugar en los motores, los cuales permiten establecer los índices previsibles del ciclo de funcionamiento de un motor de combustión interna de tipo gasodiesel $(G L P+D 2)$. El desenvolvimiento del cálculo térmico, con respecto al comportamiento del motor trabajando como gasodiesel, es validado y contrastado con los valores experimentales, mediante un análisis comparativo de las características del motor Diesel Perkins, obtenidas en el banco de pruebas Newage y las características del motor simuladas; mediante el programa computacional AJESA 2004, luego se realiza la contrastación de cada subhipótesis permitiendo así la formulación de conclusiones.
\end{abstract}

Palabras clave.- Gasodiesel, Cálculo térmico, Simulación, Banco de pruebas, Ajesa 2004, Newage, Válvula RND, Características de velocidad, Características de carga, Análisis comparativo.

\begin{abstract}
This research was conducted in Lima and is on the development and implementation of a program based on thermal calculation; to determine the operating parameters of an internal combustion engine for automotive use, by simulating various processes occurring in engines, which allow to establish the expected rate of the operating cycle of an internal combustion engine of gasodiesel type $(G L P+D 2)$. The development of the thermal calculation, based on the behavior of the engine working as gasodiesel, is validated and contrasted with the experimental values, through a comparative analysis of the characteristics of the diesel engine Perkins, obtained in the test Newage and simulated characteristics of the engine; by the computer program AJESA 2004, finally each sub-hypothesis is tested and this allows us to formulate conclusions.
\end{abstract}

Key words.- Gasodiesel, Thermal calculation, Simulation, Testing bench, Ajesa 2004, Newage, RND valve, Speed characteristics, Load characteristics, Comparative analysis.

\footnotetext{
${ }^{1}$ Tesista egresado de la Escuela de postgrado de la Facultad de Ingeniería Mecánica de la Universidad Nacional de Ingeniería, ${ }^{2}$ Phd. Docente principal e investigador de la Facultad de Ingeniería Mecánica de la Universidad Nacional de Ingeniería.
} 


\section{INTRODUCCIÓN}

En la actualidad existe dificultad en talleres y centros de servicio automotriz para poder determinar los parámetros de funcionamiento de un motor de combustión interna, ya que la gran mayoría de estos no cuentan con un banco de pruebas dentro de sus instalaciones, en ese sentido proponemos la elaboración de un software en base al cálculo térmico para poder determinarlos y predecir el funcionamiento de un MCI- Diesel Perkins, modelo CV246 gasodiesel (GLP+D2) y esto nos va a permitir, desarrollar una metodología que permita caracterizar un motor diésel a partir de los datos del catálogo del fabricante y luego estudiar los parámetros fundamentales de los motores gasodiesel, sus procesos de trabajo, y sus características.

El objetivo general del trabajo de investigación es determinar el funcionamiento de un motor de combustión interna Diesel Perkins CV146 gasodiesel utilizando un programa computacional basado en el cálculo térmico; sin utilizar un banco de pruebas y comprobarlos mediante un análisis comparativo de las curvas características. Para lo cual vamos a elaborar y desarrollar un programa computacional (software), para simular, visualizar y programar equipos o instalaciones costosas, que permitan mejorar el proceso de investigación, hacer posible el ensayo de motores no disponibles en el laboratorio y posibilitar el ensayo "personal" y eliminar las limitaciones de tiempo de ensayo a pie de máquina, determinar las ventajas del uso del sistema GLP-Diesel-RND, mediante la realización de un análisis comparativo entre los datos teóricos obtenidos luego de la aplicación del programa de cómputo en Visual Fox Pro AJESA 2004, con los datos experimentales del funcionamiento del motor gasodiesel, y observar las mejoras que se producen.

\section{CÁLCULO TÉRMICO MEDIANTE MODELO MATEMÁTICO DEL MOTOR DIESEL PERKINS SOBREALIMENTADO USANDO GLP+D2}

\section{Definición}

Se llama cálculo térmico; a la determinación de los parámetros de los procesos del ciclo del motor, es decir, es la simulación matemática de la operación del motor mediante cálculo.

El cálculo térmico permite, también, determinar las dimensiones principales del motor. Para realizar el cálculo térmico se requiere conocer:

- Los datos técnicos del motor, proporcionados por el fabricante.

- Las condiciones ambientales a las que operará, se tomará como base normas para establecer la presión y temperatura (según la norma británica BS AV 14 1971, Po= $0,1 \mathrm{MPa}$ y $\mathrm{T}_{0}=20^{\circ} \mathrm{C}$.

Cálculo térmico de un motor a gas de encendido por compresión de cuatro tiempos

Finalidad.- El cálculo térmico de los motores a gas tiene la finalidad de determinar analíticamente los parámetros energéticos del motor.

En la metodología propuesta, los cálculos corresponden al régimen nominal de velocidad del motor y a plena carga, es decir, el régimen donde la cremallera de la bomba está en su posición de suministro máximo.

\section{Cálculo térmico de un motor gasodiesel}

Para el cálculo térmico se plantea las siguientes condiciones:
a) El motor será de formación interna de la mezcla GLP+D2, en diferentes proporciones.
b) El combustible gaseoso empleado es el gas licuado de petróleo, en mezcla con el petróleo diesel 2.
c) Se mantiene la relación de compresión del motor original.
d) El cálculo térmico se realizará para el régimen nominal del motor. e) Para el ingreso del GLP se utilizará la
válvula RND. 
Tabla 1. Datos técnicos del motor Diesel.

\begin{tabular}{|c|c|}
\hline $\begin{array}{c}\text { Datos Técnicos del Motor } \\
\text { Diesel }\end{array}$ & Valores \\
\hline Marca & PERKINS \\
\hline Modelo & $\mathrm{C} 4.236 . \mathrm{V}$ \\
\hline Número de tiempos & 4 \\
\hline Relación de comprensión & 16 \\
\hline Potencia del motor $\mathrm{NE}(\mathrm{kW})$ & 61 \\
\hline Cilindrada total Vh (litros) & 3,86 \\
\hline Carrera de pistón $\mathrm{S}(\mathrm{mm})$ & 127 \\
\hline Diámetro de pistón D (mm) & 98,43 \\
\hline $\begin{array}{l}\text { Frecuencia de rotación del eje } \\
\text { cigüeñal n (rpm) }\end{array}$ & 2800 \\
\hline Número de cilindros z & 4 \\
\hline $\begin{array}{l}\text { Consumo específico efectivo } \\
\text { de combustible ge }(\mathrm{kg} / \mathrm{kW}-\mathrm{h})\end{array}$ & - \\
\hline $\begin{array}{l}\text { Velocidad media del pistón } \\
\mathrm{vp},(\mathrm{m} / \mathrm{s})\end{array}$ & - \\
\hline $\begin{array}{l}\text { Diámetro de la garganta de la } \\
\text { válvula de admisión Ds. (m) }\end{array}$ & 0,041 \\
\hline $\begin{array}{l}\text { Alzada de válvula, longitud } \\
\text { por resorte exterior }\end{array}$ & 0,0178 \\
\hline Longitud de biela 1 (m) & 0,2191 \\
\hline $\begin{array}{l}\text { Tipo de cámara de } \\
\text { Combustión }\end{array}$ & $\begin{array}{l}\text { Inyección } \\
\text { directa }\end{array}$ \\
\hline $\begin{array}{l}\text { Radio de la manivela } \mathrm{R} \text {, } \\
(\mathrm{mm})\end{array}$ & 63,5 \\
\hline
\end{tabular}

Tabla 2. Datos de partida para el cálculo térmico.

\section{Datos de Partida para el Cálculo Valores Térmico}

\begin{tabular}{|c|c|}
\hline \multicolumn{2}{|l|}{ Combustibles empleados: } \\
\hline \multicolumn{2}{|l|}{ - GLP, compuesto de \% de volumen } \\
\hline$-\mathrm{C}_{4} \mathrm{H}_{10}$ & 50 \\
\hline$-\mathrm{C}_{3} \mathrm{H}_{8}$ & 50 \\
\hline$-\mathrm{N}_{2}$ & 0 \\
\hline - Densidad de GLP $\mathrm{p}_{\text {gas, }} \mathrm{kg} / \mathrm{m}^{3}$ & 559 \\
\hline \multicolumn{2}{|l|}{$\begin{array}{l}\text { - Petróleo diésel compuesto de, en \% } \\
\text { de masa }\end{array}$} \\
\hline$-\mathrm{C}$ & 0,870 \\
\hline$-\mathrm{H}$ & 0,126 \\
\hline$-\mathrm{O}$ & 0,004 \\
\hline$-S$ & 0,005 \\
\hline$-\mathrm{W}$ & 0,00005 \\
\hline Densidad pdiesel, $\mathrm{kg} / \mathrm{m}^{3}$ & 862 \\
\hline $\begin{array}{l}\text { Cantidad de combustible diésel } \\
\text { empleado en la mezcla en el régimen } \\
\text { nominal en }(\%)\end{array}$ & 60 \\
\hline $\begin{array}{l}\text { Cantidad de combustible GLP empleado } \\
\text { en la mezcla en el régimen nominal en } \\
(\%)\end{array}$ & 40 \\
\hline \multicolumn{2}{|l|}{ Condiciones ambientales } \\
\hline - Presión atmosférica $\mathrm{p}_{\mathrm{o}}, \mathrm{MPa}$ & 0,1033 \\
\hline - Temperatura atmosférica $\mathrm{T}_{\mathrm{o}}, \mathrm{K}$ & 293,00 \\
\hline \multicolumn{2}{|l|}{ Condiciones de intercambio de gases } \\
\hline - Presión de los gases residuales $\mathrm{p}_{\mathrm{r}, \mathrm{MPa}}$ & 0,112 \\
\hline $\begin{array}{l}\text { - Temperatura de los gases residuales } \mathrm{T}_{\mathrm{r}} \text {, } \\
\mathrm{K}\end{array}$ & 760 \\
\hline - Calentamiento de la carga $\Delta \mathrm{T}, \mathrm{K}$ & 10 \\
\hline \multicolumn{2}{|l|}{$\begin{array}{l}\text { Condiciones de la formación de la } \\
\text { mezcla }\end{array}$} \\
\hline $\begin{array}{l}\text { - Coeficiente de exceso de aire en el } \\
\text { régimen nominal }\end{array}$ & 1,8 \\
\hline \multicolumn{2}{|l|}{$\begin{array}{l}\text { Condiciones de los procesos de } \\
\text { compresión, expansión y combustión }\end{array}$} \\
\hline $\begin{array}{l}\text { - Índice politrópico de compresión } \mathrm{n}_{1}= \\
(1,33 \div 1,37)\end{array}$ & 1,332 \\
\hline $\begin{array}{l}\text { - Îndice politrópico de expansión } \mathrm{n}_{2}= \\
(1,20 \div 1,27)\end{array}$ & 1,192 \\
\hline $\begin{array}{l}\text { - Coeficiente de aprovechamiento de } \\
\text { calor en el punto z del diagrama } \\
\text { indicado } \xi z\end{array}$ & 0,87 \\
\hline \multicolumn{2}{|l|}{ Condiciones de los parámetros indicados } \\
\hline $\begin{array}{l}\text { - Coeficiente de plenitud del diagrama } \\
\text { indicado } \varphi d\end{array}$ & 0,96 \\
\hline & 1,8 \\
\hline \multicolumn{2}{|l|}{ - Coeficiente de exceso de aire ${ }^{a}$} \\
\hline - TTk & 1,15 \\
\hline
\end{tabular}




\section{Instalación del programa de cálculo térmico "AJESA2004"}

Para proceder a la instalación del programa, seguir los pasos que a continuación se indican:

Contar con el CD de instalación el cual debe contar con los siguientes archivos o carpetas.

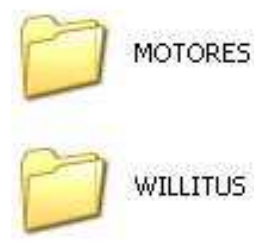

Al abrir la carpeta Willitus encontraremos lo siguiente.

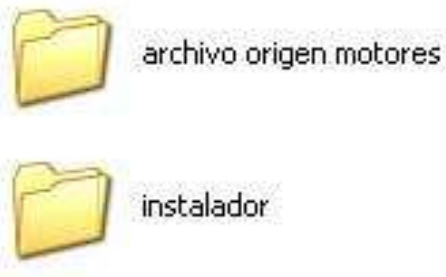

Al abrir la carpeta instalador verificamos que contengan los siguientes archivos

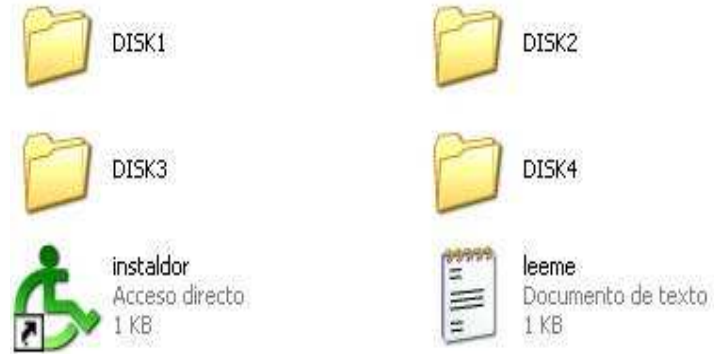

Copiamos esta carpeta denominada instalador en el disco $\mathrm{C}$, o en el disco que esté trabajando, luego procedemos a la instalación haciendo doble clic o presionando enter en el icono de color verde denominado instalador, y veremos lo siguiente.

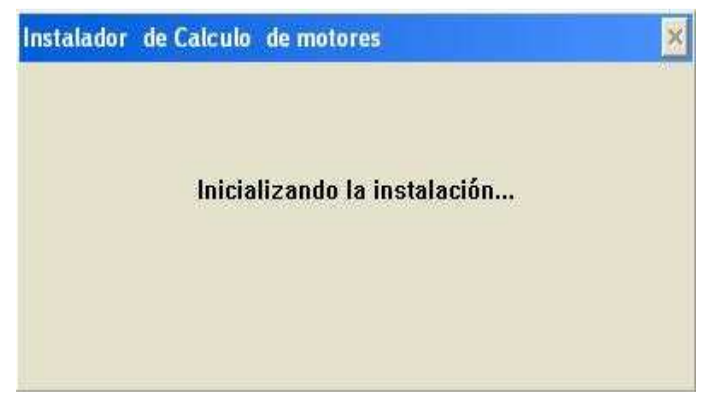

Inmediatamente aparecerá la siguiente pantalla.

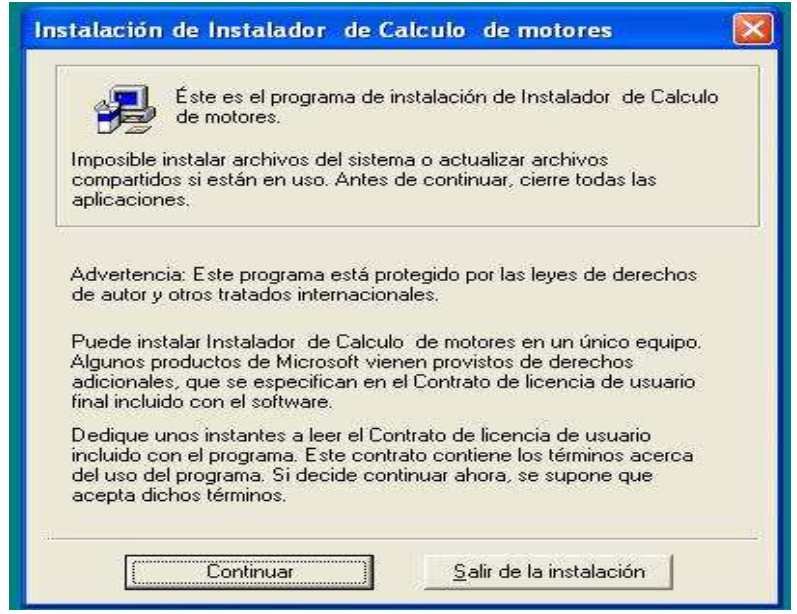

Presionamos la tecla enter en el cuadro continuar y aparecerá el siguiente recuadro.

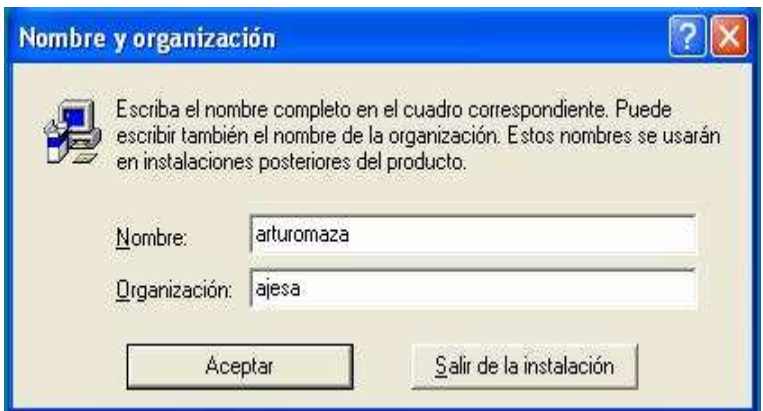

Colocamos el nombre del usuario y el nombre de la Organización y hacemos click en aceptar. Una vez confirmada la información aparecerá el siguiente recuadro.

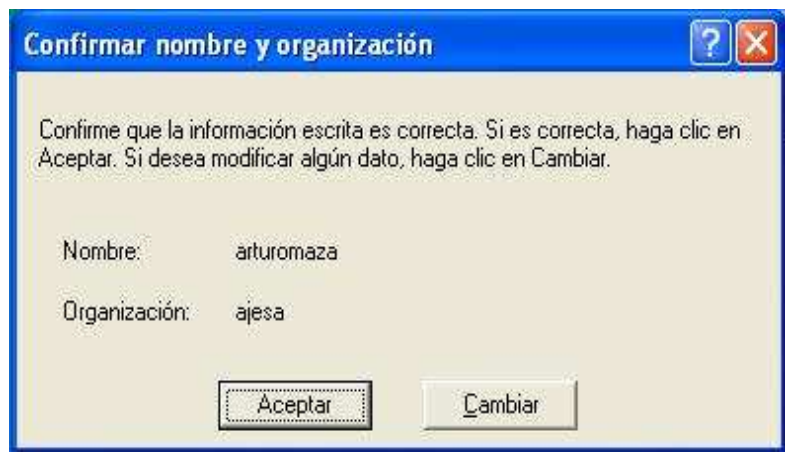

Una vez confirmado los datos hacemos click en aceptar y se completara la instalación presionando enter en aceptar o haciendo clic en el mismo. 


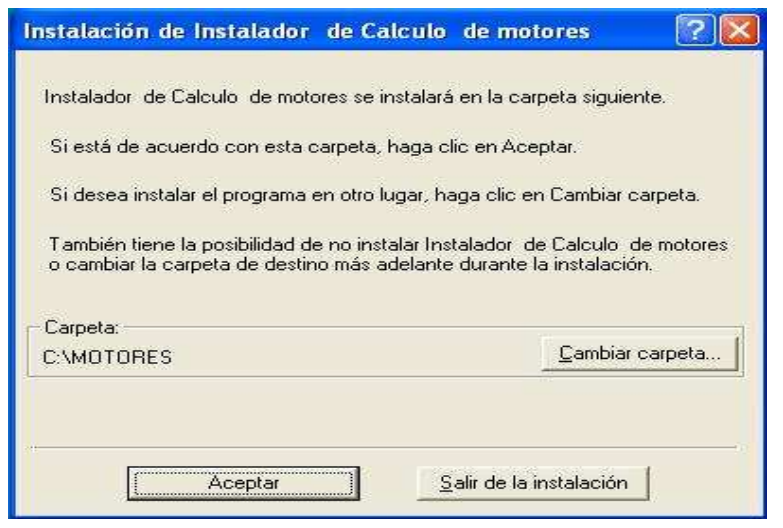

Aparecerá el cuadro donde se muestra el icono de un monitor el cual hay que presionar para culminar con la copia de los archivos al sistema.

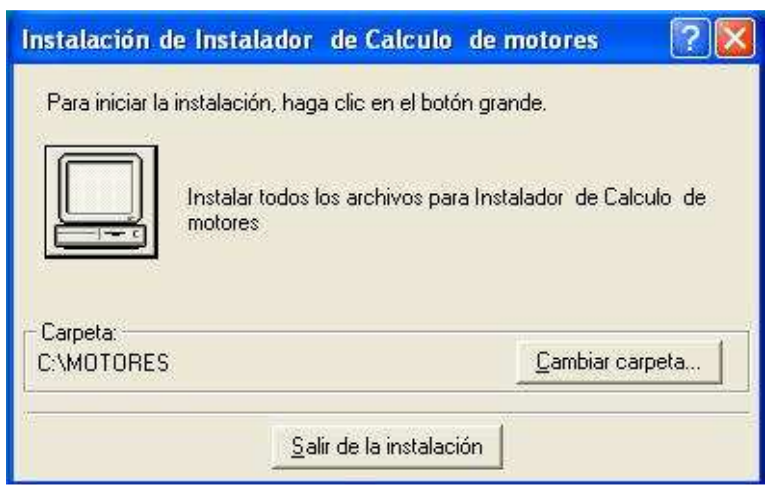

Sigue el proceso de copia de archivos en la carpeta correspondiente.

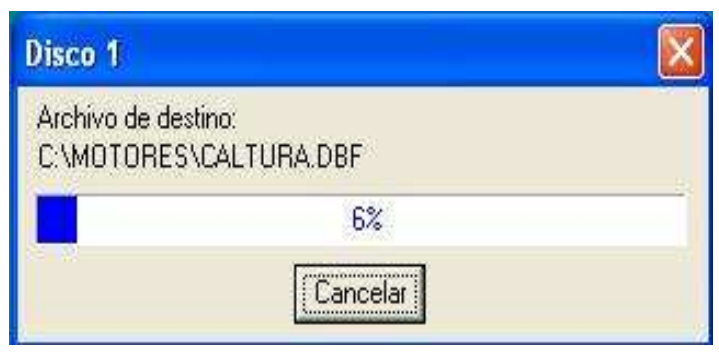

Una vez culminada la instalación con la copia de archivos, inmediatamente aparece en la pantalla la siguiente configuración con lo cual se ve que el programa se ha instalado convenientemente $y$ estamos listos para trabajar.

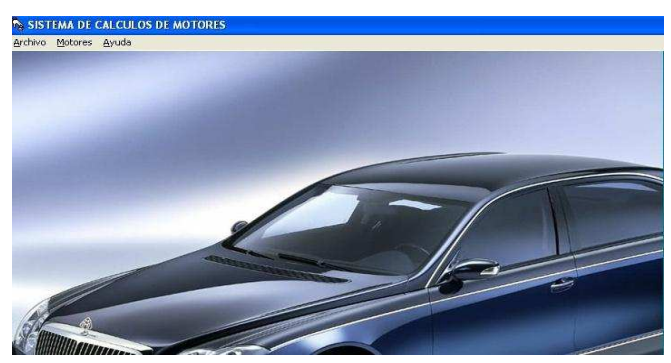

NOTA.- Culminado el proceso de instalación y con el programa ya en el sistema, para poder trabajar con mayor comodidad y ejecutar el programa es necesario realizar el siguiente paso final.

Ingresamos a la segunda carpeta denominada MOTORES mostrada en el paso 1. Abrimos haciendo doble clic y verificamos la existencia del icono siguiente.

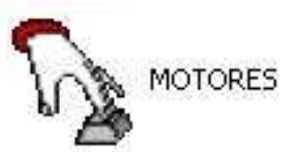

Este icono hay que enviarlo al acceso directo, para lo cual hacemos click con el botón derecho del mouse y lo enviamos al acceso directo. De esta manera podemos trabajar e ingresar al programa directamente desde el escritorio.

Así queda instalado el programa de cálculo térmico, denominado AJESA2004., y listo para su ejecución.

Fórmulas empleadas para la determinación de las características de velocidad - ambiente visual FoxPro

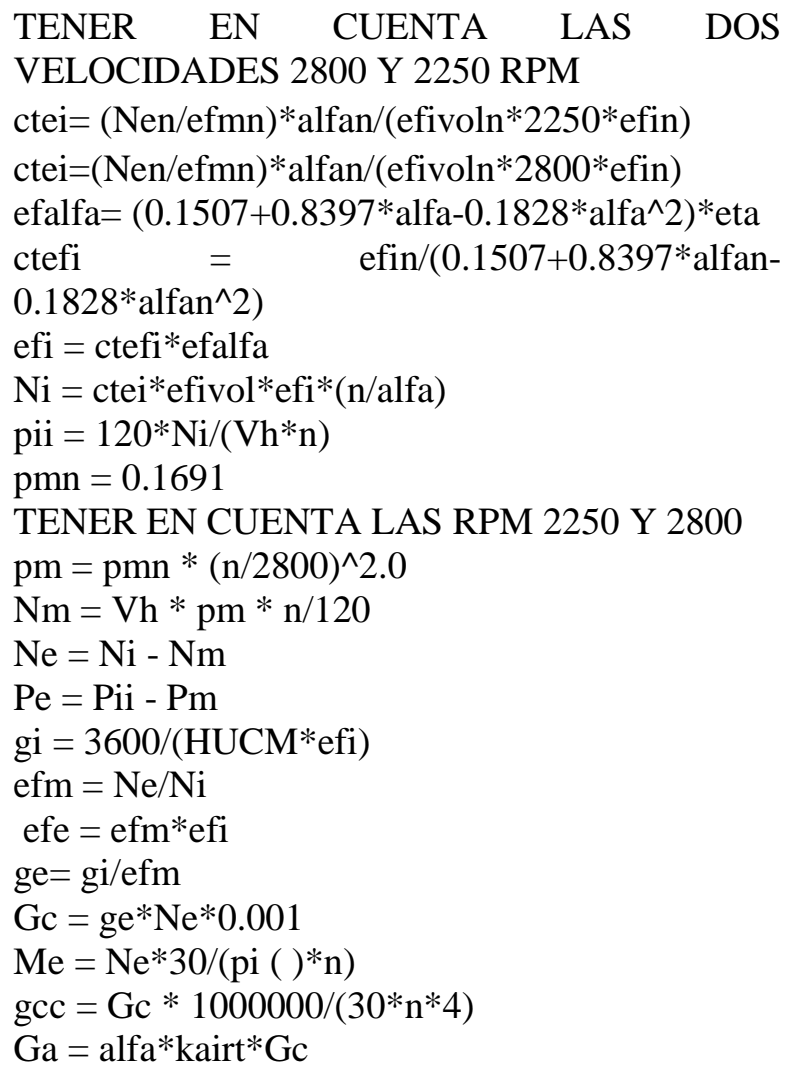


Fórmulas empleadas para la determinación de las características de carga- ambiente visual FoxPro

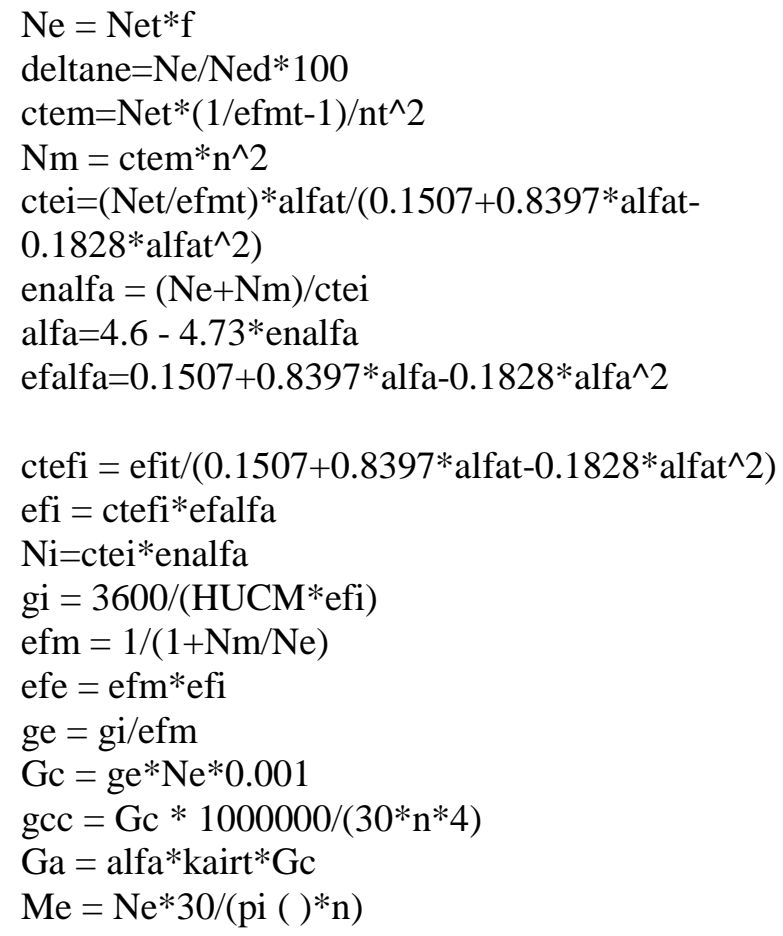

Validación del programa para un motor Diesel Perkins sobrealimentado presentación de resultados de la simulación mediante ajesa 2004 usando $100 \%$ petróleo Diesel 2, alternativa "G"

\section{Ingreso de datos de partida}

Según el procedimiento seguido por el programa, al ejecutar AJESA 10 en primer término se toma en cuenta el ingreso de los datos de partida, de acuerdo a la siguiente secuencia:

INDICAR SI YA INGRESO LOS DATOS INICIALES DEL MOTOR DIESEL PERKINS SOBREALIMENTADO, en caso que la respuesta sea SI, el programa considera los datos incluidos en él, para su ejecución, todas las condiciones establecidas para el motor del BANCO NEWAGE. En caso de que la respuesta sea "NO", inmediatamente el programa nos indica ingresar los datos de la Tabla 3: Seguidamente el programa pregunta:

A QUE CONDICIONES ATMOSFÉRICAS VA A TRABAJAR EL MOTOR AHORA INA/PERÚ/OTRO. Si la respuesta es INA, el programa continúa y pregunta. CON QUE PORCENTAJE DE COMBUSTIBLE (GLP+DIESEL2) TRABAJARA EL MOTOR $\mathrm{H} / \mathrm{E} / \mathrm{I} / \mathrm{F} / \mathrm{G}$

Es necesario mencionar que estos porcentajes de la mezcla combustible corresponden a las alternativas H: 25-75, E: 20-80, I: 15-85, F: 1090, y G: $100 \%$ en porcentajes de GLP y de petróleo DIESEL2 respectivamente, en este caso respondemos "G" es decir 100\% DIESEL2. Inmediatamente el programa empieza a trabajar y luego nos arroja los resultados de la simulación en la Tabla TRESULTADOS.

Tabla 3. Ingreso de datos de partida.

TABLA N` 5.1: INGRESO DE DATOS
\begin{tabular}{|l|c|}
\hline DELL MEDIO AMBIENTE \\
\hline TEMPERATURA $\left.{ }^{\circ} \mathrm{C}\right)$ \\
\hline PRESIÓN ( MPa) & 20 \\
\hline DEL COMBUSTIBLE & 0,1033 \\
\hline CARBONOM & 0,851 \\
\hline HIDRONENOM & 0,1464 \\
\hline OXIGENOM & 0,0024 \\
\hline AZUFREM & 0,003 \\
\hline WCM & 0,00003 \\
\hline PODER CALORIFICO DE LAMEZCLA MJ/kg & 43,60 \\
\hline DEL MOTOR & 61 \\
\hline POTENCIA NOMINAL KW & 2800 \\
\hline RPM NOMINAL & \\
\hline
\end{tabular}

\begin{tabular}{|l|c|}
\hline NUMERO DE CILINDROS & 4 \\
\hline CLLINDRADA (Vh), Lit. & 3,86 Lit \\
\hline CARRERA (mm) & 127 \\
\hline DIAMETRO (mm) & 98,43 \\
\hline DIAMMETRO DE GARGANTA (mm) & 41 \\
\hline ALZADA DE VALVULA (mm) & 17,8 \\
\hline RELACION DE COMPRESION & 16 \\
\hline LONGITUD DE BIELA (cm) & 21,91 \\
\hline TIPO DE CAMARA & NS \\
\hline
\end{tabular}

\section{Resultado de la simulación del cálculo térmico}

Los resultados de la simulación del cálculo térmico que se muestran a continuación, se han obtenido luego de ejecutar el programa principal AJESA10, según se muestra en el administrador de proyectos, del Microsoft visual FoxPro, al hacer click en todos, se muestra una base de datos, en la parte correspondiente a tablas libres, ahí encontramos en primer término la tabla denominada TRESULTADOS, de esta tabla 
mediante la aplicación del programa AJESA12, se obtiene la tabla CALTURA, posteriormente se ejecuta el programa AJESA13, y se obtiene la tabla RVELOCIDAD, luego se ejecuta el programa AJESA14 y se obtiene la tabla RCCARGA, con el programa AJESA15 se obtiene la tabla RCCARGA1, luego se ejecuta el programa AJESA16, AJESA17, AJESA18, AJESA19, en forma secuencial y obtenemos las tablas ,RCMEZCLA, RCREGULACION, RCECONOMIA, y RCECONOMIA1 respectivamente, de estas tablas vamos a considerar por su importancia para el análisis comparativo con los datos experimentales; las siguientes:

TRESULTADOS.- En esta tabla se muestran los valores de todos los parámetros del motor diesel Perkins sobrealimentado.

CALTURA.- Se muestra, los valores para las características de altura del motor. RCVELOCIDAD.- Podemos observar los valores para las características de velocidad.

RCCARGA.- Vemos los valores para las características de carga del motor.

\section{Análisis comparativo gráfico}

Para efectuar el análisis comparativo gráfico, vamos a tener en consideración las tablas correspondientes, donde se han considerado los valores tanto experimental como simulados, tanto para una mezcla de D2+GLP considerando la alternativa "E" así como para D2 alternativa "G", es importante resaltar, que en ambos casos se ha adoptado la velocidad de rotación nominal de 2250 RPM, según el protocolo de pruebas y los valores simulados, de tal manera de aproximarnos a los valores asignados en el protocolo de ensayos.

Con las tablas indicadas se elaboran los gráficos de las características de velocidad y de carga del motor en análisis, estos gráficos se muestran en las Figuras 1 al 7 , estos gráficos han sido elaborados en AUTOCAD versión 2008, en base a los datos tabulados en la hoja de cálculo Microsoft EXCEL.

\section{Características de velocidad}

I.-Análisis de las características de velocidad experimental: nnom $=2250 \mathrm{rpm}$ y características de velocidad simuladas GLP+D2 alternativa "E", nnom $=2250 \mathrm{rpm}$.

II.-Análisis de las características de velocidad experimental: $\mathrm{n}$ nom $=2250 \mathrm{rpm}$ y características de velocidad simuladas $100 \%$ D2 alternativa "G", n nom=2250 rpm.

\section{Características de carga}

III.- Análisis características de carga experimental nnom $=2250$ y características de carga simuladas GLP+D2 "E", para nnom=2250.

- Características de carga experimental para nnom $=2250 \mathrm{rpm}$ y simulada con nnom $=2250$ rpm, utilizando GLP+D2 "E", n = 1250 rpm.

- Características de carga experimental para nnom $=2250 \mathrm{rpm}$ y simulada con $\mathrm{nnom}=2250$ rpm, utilizando GLP+D2 "E", $\mathrm{n}=1500 \mathrm{rpm}$.

- Características de carga experimental para nnom $=2250 \mathrm{rpm}$ y simulada con $\mathrm{nnom}_{\text {nom }}=2250$ rpm utilizando GLP+D2 "E", $n=2000$ rpm.

- Características de carga experimental para nnom $=2250$ y simulada con nnom $=2250 \mathrm{rpm}$ utilizando GLP+D2 "E", n = 2250 rpm.

IV.- Análisis características de carga experimental nnom $=2250 \mathrm{rpm}$ y características de carga simuladas $100 \%$ D2 "G", para $n$ nom $=2250 \mathrm{rpm}$

- Características de carga experimental para $\mathrm{n}_{\text {nom }}=2250 \mathrm{rpm}$ y simulada con $\mathrm{n}_{\text {nom }}=2250$ rpm utilizando 100\% D2 "G", n = 1500 rpm.

- Características de carga experimental para $\mathrm{n}_{\text {nom }}=2250 \mathrm{rpm}$ y simulada con $\mathrm{n}_{\text {nom }}=2250$ rpm utilizando 100\%D2 "G", $\mathrm{n}=2000 \mathrm{rpm}$.

- Características de carga experimental para $\mathrm{n}_{\text {nom }}=2250 \mathrm{rpm}$ y simulada $\mathrm{n}_{\text {nom }}=2250 \mathrm{rpm}$ utilizando 100\%D2 "G", $\mathrm{n}=2250 \mathrm{rpm}$.

- Características de carga experimental para $\mathrm{n}_{\text {nom }}=2250 \mathrm{rpm}$ y simulada $\mathrm{n}_{\text {nom }}=2250 \mathrm{rpm}$ utilizando 100\%D2 "G", n = $2400 \mathrm{rpm}$. 

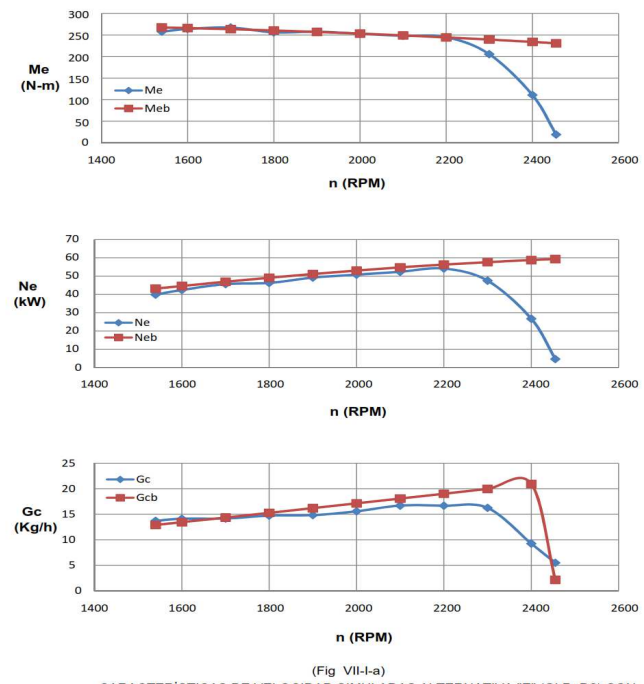

CARACTERISTICAS DE VELOCIDAD SIMULADAS ALTERNATIVA "E" (GLP+D2) CON
NNOm: 2800 RPM Y CARACTERISTICAS DE ENSAYO CON N NOM" 2250 RPM (GLP+D2)

MAZA001YA

Fig. 1 Características de velocidad simuladas alternativa " $E$ ", $\mathbf{M e}, \mathbf{N e}, \mathbf{G c}$, con Nnom $2800 \mathrm{rpm}, \mathrm{y}$ características de ensayo con Nnom $=2250 \mathrm{rpm}$.

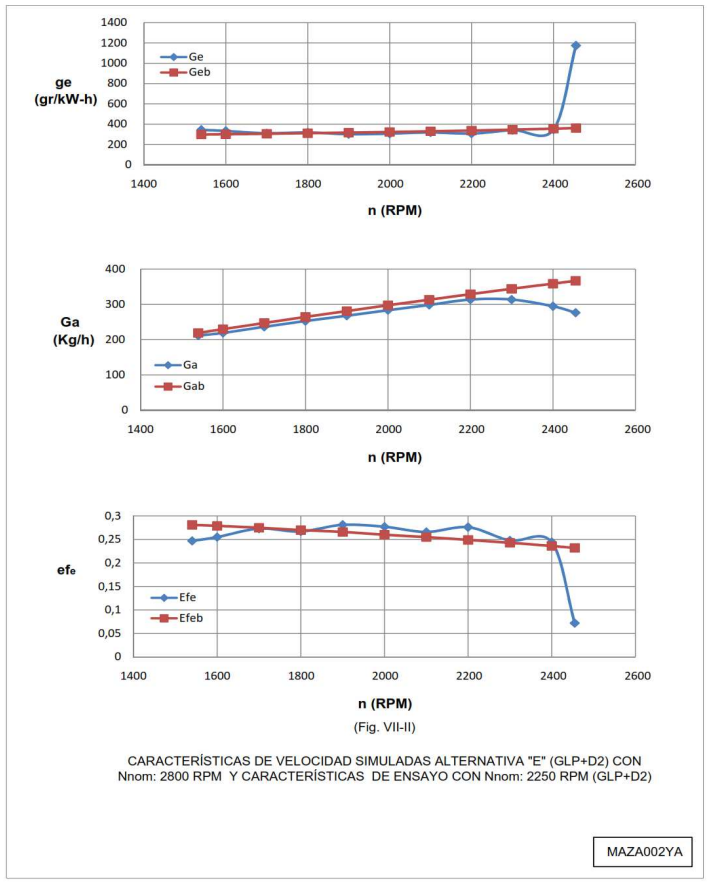

Fig. 2 Características de velocidad simuladas alternativa " $E$ ", ge, $\boldsymbol{G a}$, efe, con $N_{\text {nom }}$ 2800 rpm, y características de ensayo con $N_{\text {nom }}=2250 \mathrm{rpm}$.

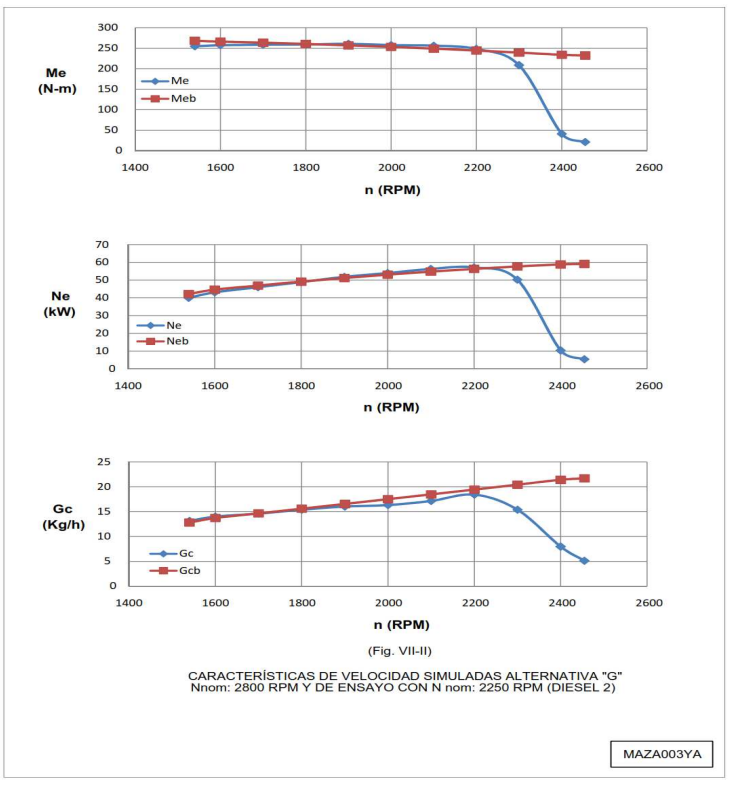

Fig. 3 Características de velocidad simuladas alternativa " $G$ ", $\mathrm{Me}, \mathrm{Ne}, \mathrm{Gc}$, con $\mathrm{N}_{\text {nom }}$ 2800 rpm, y características de ensayo con $N_{\text {nom }}=2250 \mathrm{rpm}$. (Diesel 2).

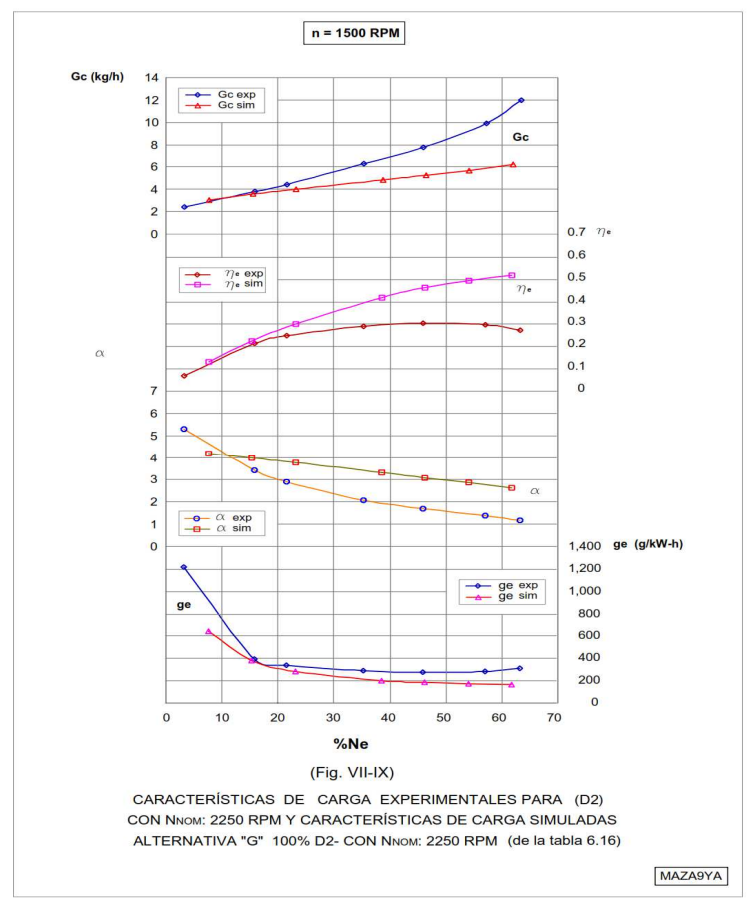

Fig. 4 Características de carga simuladas alternativa " $G$ ", con $N_{\text {nom }} 2250$ rpm, $y$ características de ensayo con $N_{\text {nom }}=2250 \mathrm{rpm}$. con $n=1500 \mathrm{rpm}$. 


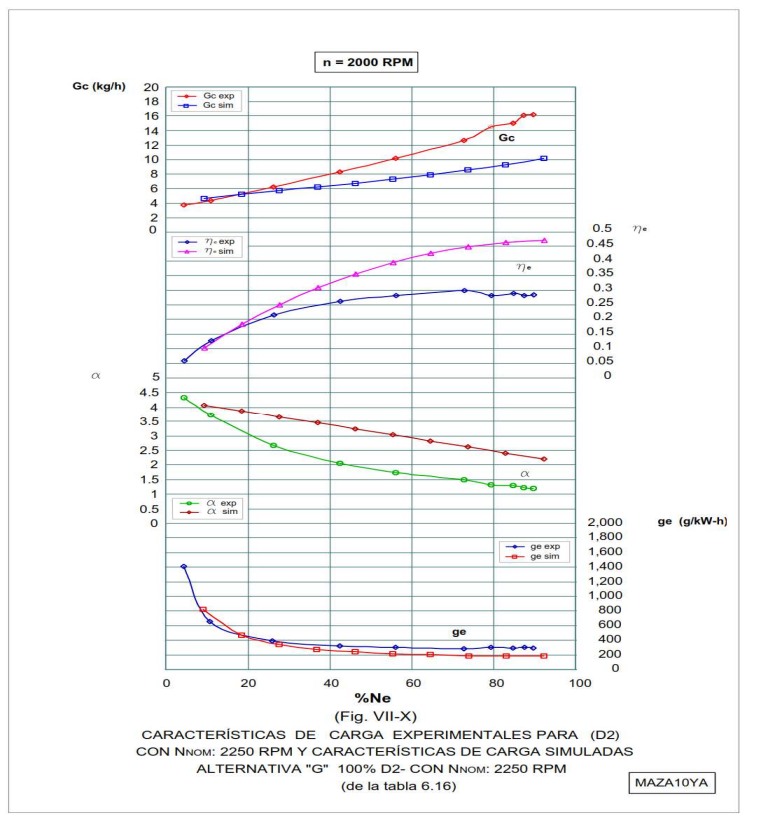

Fig. 5 Características de carga simuladas alternativa " $G$ ", con $N_{\text {nom }} 2250 \mathrm{rpm}, \mathrm{y}$ características de ensayo con $N_{\text {nom }}=2250 \mathrm{rpm}$., con $n=2000 \mathrm{rpm}$.

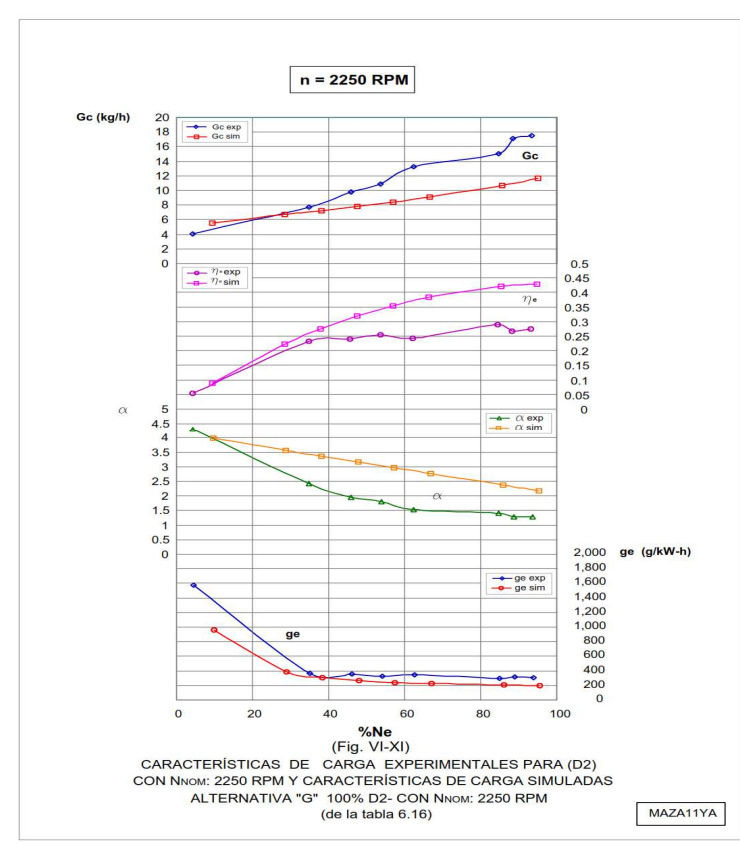

Fig. 6 Características de carga simuladas alternativa " $G$ ", con $N_{\text {nom }} 2250 \mathrm{rpm}, y$ características de ensayo con $N_{\text {nom }}=2250 \mathrm{rpm}$., con $n=2250 \mathrm{rpm}$.

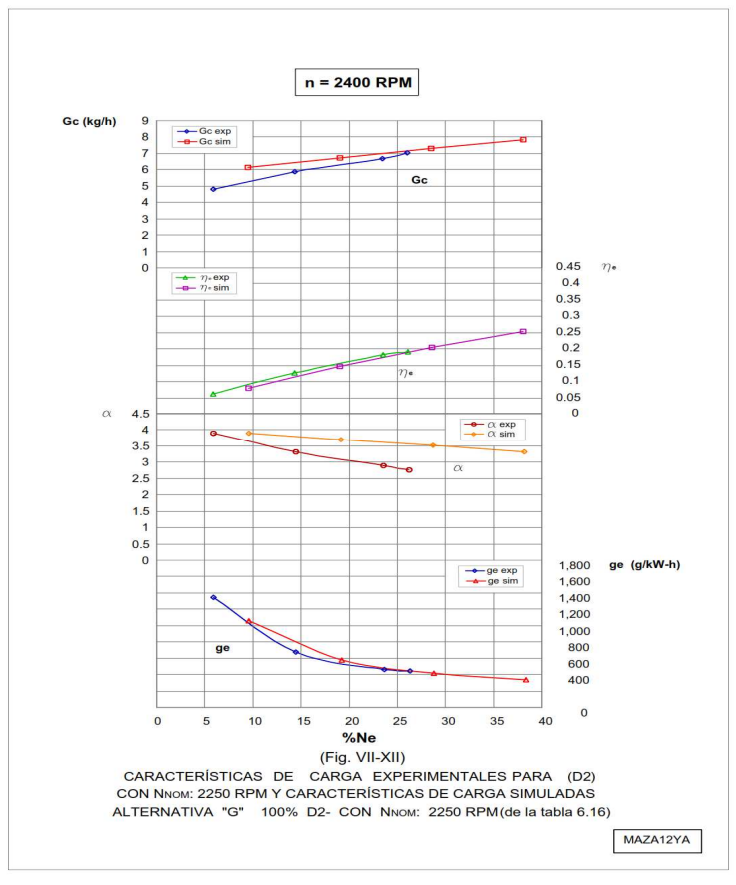

Fig. 7 Características de carga simuladas alternativa " $G$ ", con $N_{\text {nom }} 2250 \mathrm{rpm}, y$ características de ensayo con $N_{\text {nom }}=2250 \mathrm{rpm}$., con $n=2400 \mathrm{rpm}$.

\section{Discusión de resultados y contrastación de hipótesis.}

Del análisis de las curvas expuestas, se deduce dos aspectos fundamentales:

Que mediante el uso apropiado del programa de cálculo térmico, el cual incluye cálculos reiterativos en base a un programa de computo denominado Ajesa 2004, se puede predecir el funcionamiento de un motor de combustión interna y en base a este cálculo, se puede verificar si el procedimiento experimental se desarrolla o no adecuadamente mediante la contratación de los datos experimentales y simulados; para lo cual es recomendable que el equipo o banco de pruebas debe estar calibrado adecuadamente y en buen estado operativo para que los datos obtenidos sean confiables y no escapen demasiado de los valores considerados adecuados, esto se comprueba con los valores obtenidos en las tablas de variación porcentual, en estos cuadros se observa la variación porcentual para la media y media ponderada para las características de velocidad y la media para las características de carga, en ambos casos y al final de estos cuadros indicamos con las letras A de adecuado y NA de 
no adecuado para cada variación obtenida.

Es importante mencionar que según el análisis de la variación porcentual, los datos obtenidos experimentalmente que fueron extraídos de [9], en algunos casos son excesivos y en otros son menores a los esperados, por tal motivo los porcentajes de variación que son elevados y los hemos calificado como no adecuados, en base a las Tablas 4 y 5., dentro de los parámetros que se consideran aceptables y con porcentajes de variación confiables son:

Para las características de velocidad $\mathrm{Me}, \mathrm{Ne}, \mathrm{Ga}$, $\propto$, y $\eta_{e}$, para las características de carga Me, Gc, $\mathrm{Ga}, \mathrm{ge}, \mathrm{y} \eta_{\mathrm{e}}$, siendo estos: El momento efectivo (Me en $\mathrm{Nm}$ ), la potencia efectiva, ( $\mathrm{Ne}$ en $\mathrm{kW}$ ), consumo de aire $(\mathrm{Ga}$ en $\mathrm{kg} / \mathrm{h})$, coeficiente de exceso de aire $(\propto)$, consumo de combustible (Gc en $\mathrm{kg} / \mathrm{h}$ ), eficiencia efectiva $\left(\eta_{\mathrm{e}}\right)$ y consumo especifico efectivo de combustible ( $\mathrm{g}$ en $\mathrm{g} / \mathrm{kW}-\mathrm{h}$ ).

Tabla 4. Porcentaje de variación media y ponderada, de las características de velocidad $y$ de carga, alternativa " $E$ ".

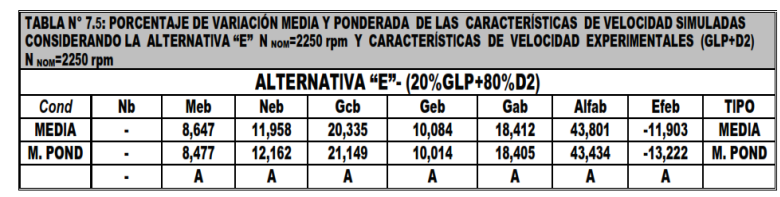

\begin{tabular}{|c|c|c|c|c|c|c|c|c|}
\hline \multicolumn{9}{|c|}{ 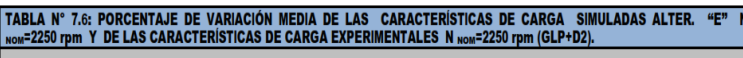 } \\
\hline \multicolumn{9}{|c|}{ Nb: 1250 RPM } \\
\hline TIPO & Meb & $\% \mathrm{Ne}$ & Gcb & Geb & Gab & Alfab & Efeb & TIPO \\
\hline \multirow[t]{3}{*}{ MEDIA } & $-4,195$ & * & -45.438 & -50.756 & 7.019 & 31.173 & 25.712 & MEDIA \\
\hline & A & - & A & NA & A & A & A & \\
\hline & $\downarrow$ & $*$ & $\downarrow$ & $\downarrow$ & $\uparrow$ & $\uparrow$ & $\uparrow$ & \\
\hline \multicolumn{9}{|c|}{$\mathrm{Nb}: 1500 \mathrm{RPM}$} \\
\hline TIPO & Meb & $\% \mathrm{Ne}$ & Gcb & Geb & Gab & Alfab & Efeb & TIPO \\
\hline \multirow[t]{3}{*}{ MEDIA } & $-4,195$ & * & -43.139 & -44.518 & 10.633 & 30.642 & 22.508 & MEDIA \\
\hline & A & - & A & A & A & A & A & \\
\hline & $\downarrow$ & $*$ & $\downarrow$ & $\downarrow$ & $\uparrow$ & $\uparrow$ & $\uparrow$ & \\
\hline \multicolumn{9}{|c|}{ Nb: 2000 RPM } \\
\hline TIPO & $\mathrm{Me}$ & $\% \mathrm{Ne}$ & $G_{c}$ & ge & Ga & a & $\eta e$ & TIPO \\
\hline \multirow[t]{3}{*}{ MEDIA } & $-4,191$ & * & -11.125 & -25.017 & 17.171 & 20.580 & 9.789 & MEDIA \\
\hline & A & - & A & A & A & A & A & \\
\hline & $\downarrow$ & * & $\downarrow$ & $\downarrow$ & $\uparrow$ & $\uparrow$ & $\uparrow$ & \\
\hline \multicolumn{9}{|c|}{$\mathrm{Nb}: 2250 \mathrm{RPM}$} \\
\hline TIPO & Meb & $\% \mathrm{Ne}$ & Gcb & Geb & Gab & Alfab & Efeb & TIPO \\
\hline \multirow[t]{3}{*}{ MEDIA } & $-4,193$ & $*$ & 0,936 & $-10,395$ & 20,397 & 12,279 & $-5,709$ & MEDIA \\
\hline & A & . & A & A & A & A & A & \\
\hline & $\downarrow$ & $*$ & $\uparrow$ & $\downarrow$ & $\uparrow$ & $\uparrow$ & $\uparrow$ & \\
\hline
\end{tabular}

Tabla 5. Porcentaje de variación media y ponderada, de las características de velocidad y de carga, alternativa "G".

\begin{tabular}{|c|c|c|c|c|c|c|c|c|c|}
\hline \multicolumn{10}{|c|}{ 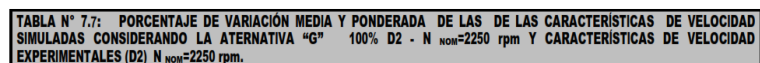 } \\
\hline \multicolumn{10}{|c|}{ ALTERNATIVA "G"- (100\%D2) } \\
\hline Cond & Nb & Meb & Neb & Gcb & Geb & Gab & Alfab & Efeb & TIPO \\
\hline MEDIA & - & 8,346 & 8,353 & 19,685 & 12,010 & 17,874 & 43,957 & $-13,628$ & MEDIA \\
\hline \multirow[t]{2}{*}{ M.POND } & . & 7,962 & 7,969 & 20,419 & 13,232 & 17,808 & 43,362 & $-15,195$ & M.POND \\
\hline & . & A & A & A & A & A & A & A & \\
\hline
\end{tabular}

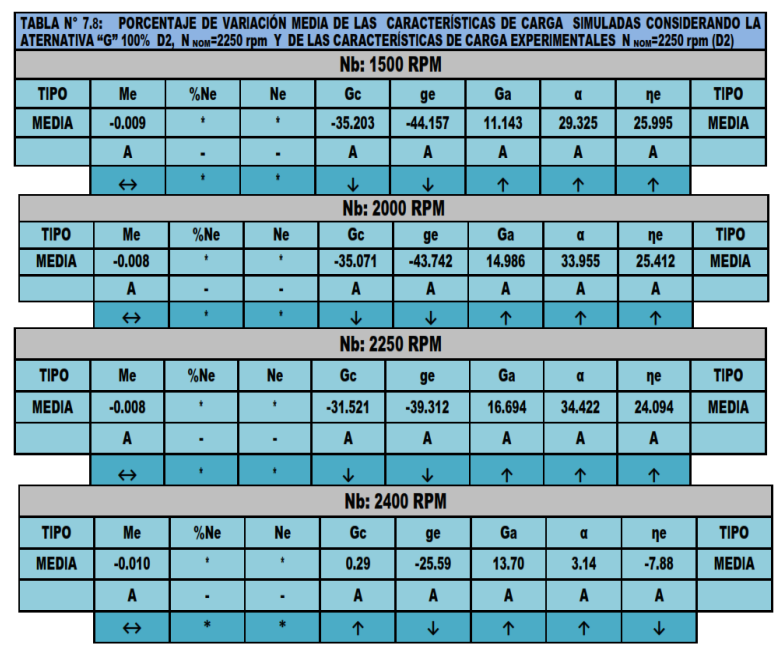

\section{CONCLUSIONES}

Debemos concluir que, mediante el uso de este programa de cálculo térmico podemos determinar mediante simulación las características más importantes de un motor Diesel Perkins, y realizando cambios en cuanto a las características del tipo de motor considerado, podremos obtener resultados satisfactorios para otro tipo de motor en base a este procedimiento. Los resultados de la simulación obtenidos y mostrados en las tablas, nos indican que en el caso del motor Diesel Perkins empleado en este trabajo, se consigue el mayor suministro de GLP cuando el motor funciona con bajas cargas y altas velocidades lo cual se corrobora en los gráficos experimentales. La utilización del GLP como combustible alternativo, demuestra su eficacia ya que disminuye el humeado del motor. Esta ventaja combinada con el mejoramiento del consumo de combustible, permite concluir que es posible mejorar los índices energéticos y de toxicidad del motor Diesel sin recurrir a modificaciones sustantivas del mismo, es decir instalando un 
dispositivo de bajo costo y fácil fabricación. Del análisis comparativo se desprende, que el programa AJESA2004 funciona adecuadamente con un margen mínimo de error. Con este programa, se puede obtener las características de un motor diesel Perkins a diferentes condiciones de funcionamiento y con diferentes proporciones de combustible (GLP+DIESEL2). Mediante la simulación con AJESA2004, se puede predecir el funcionamiento de un motor diesel turboalimentado para diferentes alturas a nivel del mar.

\section{REFERENCIAS}

1. LIRA CACHO G., "Inyección de gas licuado de petróleo en los motores diesel", Revista Tecnia, Vol. 6, Nº1, pp. 41-47, Universidad Nacional de Ingeniería, Lima, Nov. 1996.

2. Patrakhaltsev, N. N., "Cálculo térmico del motor de combustión interna" Ed. Universidad de la Amistad entre los pueblos. Moscú 1981.

3. Estrella Camacuari., "Empleo de Gas Licuado de Petróleo en un motor Diesel multicilindrico". Tesis profesional, LimaPerú.

4. Aznualdo, R., "Mejoramiento de los índices económicos y ecológicos de los motores diesel mediante la adición de gas licuado de petróleo" Tesis-UNI.

5. Vera Becerra, E. "Diseño Térmico de un turbocompresor para sobrealimentar al motor Perkins modelo 63544 para un incremento de potencia de 50\%" TesisUNI.

6. Jóvaj, M.S., "Motores de automóvil", Editorial MIR, Moscú, 1982.

7. Lastra Espinoza, L, Lira Cacho, G., Vera, Becerra, E., Experimentación y cálculo de motores de combustión interna" FIM-UNI 1990.
8. Galdós Gómez, J., "Problemas y Posibilidades de Utilización de los Combustibles Gaseosos en los Motores de Combustión Interna en el Perú". Tesis UNSAA-Arequipa, 1996.

9. Lira Cacho, G., Lastra Espinoza L., "Empleo del gas licuado de petróleo en los motores Diesel", X Congreso Nacional de Ingeniería Mecánica, Eléctrica y Ramas Afines, Lima, 1991.

10. Patrakhaltsev, N. N., Pavlokov, V. G., Oliesov, I. Y., "Nuevas Aplicaciones del gas en los Motores de Combustión Interna" UNI-FIM-IMCI. III SEMINARIO INTERNACIONAL 1996.

11. Lira Cacho, G. "Inyección de gas licuado de petróleo en los motores diésel". 'Tecnia', Vol. 6, UNI, Lima-Perú, 1996. pp. 41-47.

12. Turek, A., "Microsoft Visual FoxPro 6.0"Genere Aplicaciones de alta calidad en tiempo record. Microsoft-Corporation, publicado por MP Ediciones S.A., 2001ISBN 987974411X, pp. 344.

13. Lastra Espinoza, L., "Curso sobre Motores de Combustión Interna a Gas" Lima- Peru-2002.

14. BestReferat.ru., "El Análisis Térmico y Dinámico Del Motor de combustión interna KAMAZ-740". Trabajo de curso [799,1 $\mathrm{K}], 05 / 20 / 2011$.

15. BestReferat.ru., "Cálculo Térmico del motor D-120 y cálculo de la eficiencia operativa del tractor T-30 A". Trabajo de curso [757,9 K], 13/12/2011.

16. BestReferat.ru - Banco de resúmenes. "Cálculo del motor JAMZ-240B". Diseño térmico del motor. Trabajo práctico [187,4 $\mathrm{K}], 12 / 10 / 2009$.

Correspondencia: ing.arturomaza@gmail.com

Recepción de originales: julio 2014

Aceptación de originales: octubre 2014 